University of Nebraska - Lincoln

DigitalCommons@University of Nebraska - Lincoln

\title{
A Scalable Approach for Survivable Virtual Topology Routing in Optical WDM Networks
}

Ajay Todimala

Byrav Ramamurthy

University of Nebraska-Lincoln, bramamurthy2@unl.edu

Follow this and additional works at: https://digitalcommons.unl.edu/csearticles

Part of the Computer Sciences Commons

Todimala, Ajay and Ramamurthy, Byrav, "A Scalable Approach for Survivable Virtual Topology Routing in Optical WDM Networks" (2007). CSE Journal Articles. 85.

https://digitalcommons.unl.edu/csearticles/85

This Article is brought to you for free and open access by the Computer Science and Engineering, Department of at DigitalCommons@University of Nebraska - Lincoln. It has been accepted for inclusion in CSE Journal Articles by an authorized administrator of DigitalCommons@University of Nebraska - Lincoln. 


\title{
A Scalable Approach for Survivable Virtual Topology Routing in Optical WDM Networks
}

\author{
Ajay Todimala and Byrav Ramamurthy
}

\begin{abstract}
The survivable virtual topology routing problem is to route a virtual topology graph on a optical fiber physical topology such that the virtual topology remains connected when failures occur in the physical topology. In this work we study the problem of survivable virtual topology routing under single node/SRLG (Shared Risk Link Group) failure model. We prove that the survivable virtual topology routing problem under node/SRLG failures is NP-complete. We present an improved integer linear programming (ILP) formulation for computing the survivable routing of a virtual topology graph. However, ILP is not scalable when the network size scales more than a few tens of nodes. In this work, we present sub-classes of graphs which more accurately model an actual network and for which a survivable routing can be easily computed solving an ILP. We successfully computed the survivable routing of virtual topologies belonging to these sub-classes against link/SRLG failures for topologies of size up to 24 nodes.
\end{abstract}

Index Terms-WDM networks, shared risk link group (SRLG), survivable virtual topology routing (SVTR).

\section{INTRODUCTION}

$\mathbf{W}$ DM networks have gained tremendous popularity due to their ability to tap the enormous amount of bandwidth in an optical fiber. Their growing popularity and bandwidth capacity have made survivability in these networks an important aspect. The physical topology of a WDM network consists of nodes interconnected with one or more pairs of fiber links. The fiber links are stuffed in conduits which are laid along a right-of-way such as a railway track etc. A conduit may consist of fiber links of more than one pair of nodes. When a failure occurs in a conduit such as a conduit cut, all the fiber links in the conduit are likely to fail at the same time resulting in failure of multiple physical links (optical fiber links). So the assumption that single fiber link failures are the primary failures is no longer a good assumption in optical fiber networks. Surviving multiple link failures due to conduit cuts implies that more redundancy is required at the physical layer. However, the laying of conduits does not change over time so the sets of fiber links that are prone to failure due to conduit cuts are known ahead of time and are fixed.

The concept of Shared Risk Link Group (SRLG), a formal model to handle multiple link failures due to conduit cuts

Manuscript received February 23, 2005; revised September 12, 2006. This work was supported in part by the U. S. National Science Foundation grants (ANI-0074121 and EPS-0091900). An earlier version was presented at the International Conference on Broadband Communications, Networks and Systems (BroadNets 2004).

The authors are with the Department of Computer Science and Engineering, University of Nebraska-Lincoln, Lincoln NE 68588-0115 USA (email: \{ajayt, byrav\}@cse.unl.edu).

Digital Object Identifier 10.1109/JSAC-OCN.2007.020605. etc. was introduced in [1]. An SRLG is any sub-set of links in the network that share the risk of failing at the same time. SRLG can be used to model several types of failure conditions such as single-link failures, conduit/right-of-way failures, node failures, fiber-span failures, double-link failures or failure of any other possible subset of links sharing a common risk. A virtual topology is survivable against link/SRLG failures if all the nodes in the virtual topology remain connected. In case of node failures, a virtual topology is survivable against node failures if all the nodes in the virtual topology are connected except the node corresponding to the failed node in the physical topology. Node failures can be modeled using SRLG by grouping all the links incident on the failed node into a single SRLG.

The virtual topology routing problem is to route each logical link in the virtual topology on a path in the physical topology such that the total cost of routing all the virtual links is minimized. Often the virtual topology is different from the physical topology and may need to be reconfigured with changing traffic patterns. The wavelength assignment subproblem deals with assigning a free wavelength along the computed physical path corresponding to each virtual link in the virtual topology considering the wavelength restrictions. In this work we study how to survivably route a virtual topology against SRLG failures. Such a routing of the given virtual topology on a physical topology is called Survivable Virtual Topology Routing (SVTR).

Let us briefly survey related work on survivable virtual topology routing. The virtual topology design problem is well researched (eg., see, [3], [4], [5]) but little work has been done on the survivability aspect of the virtual topology. The design of protected virtual topologies by minimizing the number of disconnected source destination pairs due to failure of single links in the physical topology was addressed in [6]. In [7] the survivable ring virtual topology routing problem is addressed. This work presented an algorithm to find a survivable routing if one exists. In [8], [9] the survivable routing of logical topologies is considered. These works consider the addition of virtual links to make the virtual ring survivable. In [10], the issue of designing multiple protected virtual private networks (VPNs) on a single physical network is addressed. For each VPN, a working and a protection VPN are designed considering single link and node failures. But neither of the working or protection VPNs are themselves survivable. The problem of mapping a multi-graph (a graph with multiple links between any pair of nodes) Internet topology onto a physical topology for survivability against link failures was addressed in [11]. The survivable virtual topology routing 
problem against single link failures was addressed in [2] and was proved to be NP-complete. Authors presented the necessary and sufficient conditions for survivable routing and provided an ILP formulation.

In this work we study the survivable routing problem under SRLG/node failures. The rest of the paper is organized as follows. In Section II, we provide an insight into the theory of cut-sets of a graph. A cut-set of a graph is a set of edges, the removal of which disconnects the graph. A formal definition of a cut-set is provided in Section II. In Section III, based on the cut-set classification, we present the improved ILP formulation for survivable routing. The ILP formulation becomes too large to solve when the virtual topology is a general graph (even for medium-sized graphs). Therefore, in Section IV, we introduce sub-classes of graphs (to be used as virtual topologies) that have polynomial number of primary cuts. Section V presents the results from experiments of survivable routing considering different physical and virtual topologies. Section VI provides the conclusions.

\section{Survivable Routing}

In this section we formalize the survivable virtual topology routing (SVTR) problem and present the necessary and sufficient conditions. Before doing so, we present a short overview of the theory of cut-sets of a graph. In this section we also present the proof of NP-completeness of survivable routing under node failures. The necessary and sufficient conditions provide the proof of correctness of the ILP formulation for solving the SVTR problem, presented in the Section III.

\section{A. Survivable Virtual Topology Routing (SVTR)}

Let us now discuss the SVTR problem. The physical topology is represented by $G_{p}=\left(V_{p}, E_{p}\right)$ where $V_{p}$ is the set of nodes in the physical topology and $E_{p}$ is a set of bi-directional fiber links between nodes $(i, j) \in V_{p}$. A bidirectional fiber link is a pair of fiber links where each fiber is dedicated to carry data in a particular direction opposite to the other fiber. The SRLGs on the physical topology are defined by $R_{p}=\left\{r_{i} \mid r_{i}=\left\{e_{i, 1}, e_{i, 2}, \ldots e_{i, m}\right\}, 1 \leq j \leq m,\left(e_{i, j} \in E_{p}\right.\right.$ and that they share the same risk of simultaneous failure) $\}$ where $r_{i}$ is the $i^{t h}$ SRLG and $e_{i, j}$ is the $j^{t h}$ edge in the SRLG $r_{i}$. Each link in the physical topology belongs to at least one SRLG in the set $R_{p}$. This assumption is based on the fact that every physical link passes through some conduit (at least one of its own) and is a possible source of failure. To model node failures using SRLG, all the links incident on the node are grouped into a single SRLG. The virtual or logical topology is a graph $G_{l}=\left(V_{l}, E_{l}\right)$ where $V_{l} \subseteq V_{p}$ and link $(i, j) \in E_{l}$ represents logical bi-directional link between nodes $i, j \in V_{l}$. Not all the nodes in the physical topology need to be present in the virtual topology. Some of the nodes are just tapping points [12]. A tapping point is a node in the network such that it is not a source or destination for any connection request.

To route a virtual topology on a physical topology, for each link in the virtual topology, we need to find a path/route in the physical topology. Both the fiber links in the physical

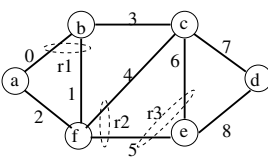

(a)

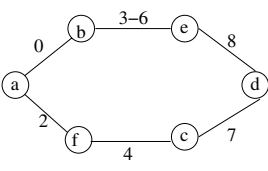

(b)

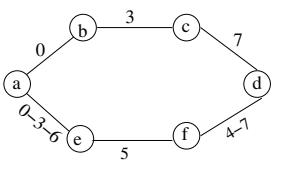

(c)
Fig. 1. (a). A 6-node physical topology with SRLG. (b). A survivable virtual topology and numbers adjacent to each virtual link show the routing. (c) A non-survivable virtual topology.

topology and logical links in the virtual topology are bidirectional. We assume that both topologies are undirected and compute a route between the given pair of nodes. The direction is immaterial as the same path can be used to route the connection in both directions.

Given the physical topology $G_{p}$, the SRLG $R_{p}$ and the virtual topology $G_{l}$ we wish to determine a routing of the virtual topology such that in the event of failure of any single SRLG in the physical topology, the virtual topology is still connected. Such a routing is called SRLG survivable routing. In the rest of the document 'survivable' refers to 'SRLG survivable' unless specified explicitly. Let us illustrate the survivable virtual topology routing problem using Fig. 1. Fig. 1(a) shows a physical topology with SRLG. Links $(a, b)$ and $(b, f)$ belong to the SRLG $r_{1}$ (indicated by dashed ovals). Similarly SRLG $r_{2}$ and $r_{3}$ containing two links each are shown in Fig. 1(a). Consider the virtual topology shown in Fig. 1(b). For each link in the virtual topology there are many different paths for routing the link on the physical topology. As shown in Fig. 1(b), the virtual link $(b, e)$ is routed on the physical links labeled 3 and 6 in the physical topology. Similarly, the physical route of other virtual links is shown in Fig. 1(b). This routing is survivable against SRLG failures. If the link $(b, e)$ of the virtual topology in Fig. 1(b) were routed on the links 1 and 5 (not shown in figure), then failure of SRLG $r_{1}$ will result in the failure of the virtual links $(a, b)$ and $(b, e)$. Therefore routing the virtual link $(b, e)$ on the links 1 and 5 results in a routing that is not survivable. Survivable routing of a virtual topology exists if there exists at least one routing of the virtual topology that is survivable. We call such a topology, survivable virtual topology. The routing of the virtual topology shown in Fig. 1(c) is not surivivable. A through examination of all possible routings of the virtual topology in Fig. 1(c), will lead to the conclusion that none of the routings of virtual topology in Fig. 1(c) on the physical topology Fig. 1(a) is survivable. Therefore a survivable routing of the virtual topology in Fig. 1(c) does not exist. Such a topology is called a non-survivable virtual topology.

\section{B. Graph Theory: Classification of Cuts of a Graph}

In this section we present an introduction to cuts of a graph and then present a classification of cuts of a graph. Given an undirected connected graph $G=(V, E)$ where $V$ is the set of nodes and $E$ is the set of edges where $(i, j)$ represents the edge between nodes $i$ and $j$. A cut $\langle S, V \backslash S\rangle$ is a partition of the set of nodes $V$ into two sub-sets $S$ and $V \backslash S$ where $V \backslash S$ represents the set $V$ minus set $S$ [13]. A cut-set $\mathcal{C S}_{G}\langle S, V \backslash S\rangle$ associated with cut $\langle S, V \backslash S\rangle$ is defined as the set of edges in 


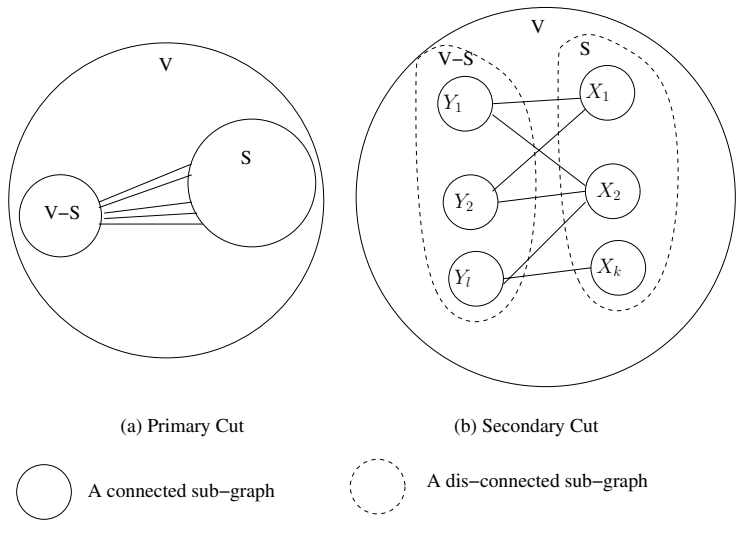

Fig. 2. Illustration of primary and secondary cuts of a graph $G=(V, E)$.

$E$ such that one end-point is in $S$ and the other is in $V \backslash S$. Thus,

$$
\mathcal{C S}_{G}\langle S, V \backslash S\rangle=\{(i, j) \mid(i, j) \in E \wedge(i \in S) \wedge(j \in(V \backslash S))\} .
$$

For any given undirected connected graph $G=(V, E)$ the number of possible cuts is $2^{n-1}$ which is exponential in terms of $n$ where $n$ is the number of nodes. The cut $\langle\{\}, V\rangle$ is the trivial cut. An induced sub-graph of a graph $G=(V, E)$ by a sub-set of nodes of $V$ is a sub-graph such that edge $(i, j)$ belongs to the sub-graph if and only if edge $(i, j)$ belongs to $G$. Let $G_{p}=\left(V_{p}, E_{p}\right)$ be the physical topology graph. $G_{p, i}=\left(V_{p, i}, E_{p, i}\right)$ is the induced sub-graph of $G_{p}$ by the node set $V_{p, i}$ where $V_{p, i}=V_{p}-\{i\}, i \in V_{p}$ and $E_{p, i}=$ $\left\{(m, n) \mid(m, n) \in E_{p} \wedge((m \neq i) \wedge(n \neq i))\right\}$.

Let us classify a cut $\langle S, V \backslash S\rangle$ of a graph into primary or secondary based on the connectedness of the partitions $S$ and $V \backslash S$ of the cut. A cut, $\langle S, V \backslash S\rangle$, is called a primary-cut if and only if both of the induced sub-graphs of $G$ by the node sets $S, V \backslash S$ are connected components. Fig. 2(a) shows a primary cut $\langle S, V \backslash S\rangle$. A cut, $\langle S, V \backslash S\rangle$, is called a secondary-cut if either of the induced sub-graphs of $G$ by the node sets $S$ and $V \backslash S$ is not a connected component. Fig. 2(b) shows a secondary cut $\langle S, V \backslash S\rangle$ with induced sub-graph $G_{S}$ consisting of $k$ connected components $G_{X_{1}}, G_{X_{2}}, \ldots, G_{X_{k}}$. The cutset associated with a primary-cut $\langle S, V \backslash S\rangle$ (correspondingly, secondary-cut) is called a primary cut-set (corrs., secondary cut-set) and represented as $\mathcal{P}\langle S, V \backslash S\rangle$ (corrs., $\mathcal{S}\langle S, V \backslash S\rangle$ ).

Theorem 1: If graph $G=(V, E)$ is connected then every secondary cut-set of $G$ is superset of at least one primary cut-set of $G$. (For proof, please refer to [17]).

\section{Necessary and Sufficient Conditions: SRLG Constraints}

Given an undirected physical topology $G_{p}$, its SRLG $R_{p}$ and a virtual topology $G_{l}$ where $V_{l} \subseteq V_{p}$. The virtual topology must be 2-edge-connected, otherwise the survivable routing does not exist. Every cut-set of the virtual topology then has at least 2 edges. If the virtual topology is only edge-connected then there exists a cut-set with only a single edge. When an SRLG that is used for routing the single edge of the cut-set fails, the virtual topology gets disconnected. Therefore, a 1edge connected virtual topology cannot be survivably routed.
The necessary condition on the physical topology for the existence of survivable routing is that the physical topology must not get disconnected due to failure of any single SRLG. If the physical topology gets disconnected due to a single SRLG failure, then it is not possible to route the virtual topology such that it remains connected. Assuming that the physical $\left(G_{p}\right)$ and virtual $\left(G_{l}\right)$ topologies satisfy the necessary conditions for the existence of survivable routing, we present the necessary and sufficient conditions for a routing of $G_{l}$ on $G_{p}$ to be survivable in Theorem 2 below.

Theorem 2: Given the physical topology $G_{p}$, SRLG set $R_{p}$ and the virtual topology $G_{l}$, the routing of $G_{l}$ over $G_{p}$ is survivable if and only if $\forall r \in R_{p}$ and for all primary cutsets $P_{G_{l}}\left\langle S, V_{l} \backslash S\right\rangle$ of the virtual topology at least one link in $P_{G_{l}}\left\langle S, V_{l} \backslash S\right\rangle$ is not routed over any of the links in $r=$ $\left\{e_{1}, e_{2}, \ldots, e_{m}\right\}$. (For proof, please refer to [17]).

Theorem 3: The survivable virtual topology routing (SVTR) problem under SRLG failures is NP-complete. (For proof, please refer to [17]).

\section{Necessary and Sufficient Conditions: Node Failures}

Given the undirected physical topology $G_{p}$ and undirected graph virtual topology $G_{l}$. The virtual topology is 2 -vertex connected, otherwise the survivable routing does not exist. Let us first see the necessary conditions on the virtual topology for a survivable routing to exist (Lemma 4).

Lemma 4: Given the physical topology $G_{p}$ and the virtual topology $G_{l}$. if $G_{l, i}$ is not connected for some $i \in V_{p}$, then a node-survivable routing of $G_{l}$ over $G_{p}$ does not exist. (For proof, please refer to [17]).

Theorem 5 states the necessary and sufficient conditions for survivable routing of the virtual topology $G_{l}$ over the physical topology $G_{p}$ under single node failures.

Theorem 5: A routing is survivable if and only if for all $G_{l, i}, i \in V_{p}$, at least one link in $P_{G_{l, i}}\left\langle S, V_{l, i} \backslash S\right\rangle$ is not routed on edge set $\left(E_{p} \backslash E_{p, i}\right)$ where $E_{p, i}$ is the edge set of induced sub-graph $G_{p, i}=\left(V_{p, i}, E_{p, i}\right)$ of $G_{p}$. (For proof, please refer to [17]).

Theorem 6: Survivable virtual topology routing (SVTR) problem under node failures is NP-complete. (For proof, please refer to [17]).

\section{INTEGER LiNEAR PROGRAMMING FORMULATION}

Given the undirected physical topology $G_{p}=\left(V_{p}, E_{p}\right)$, the SRLG set $R_{p}$ and the virtual topology $G_{l}=\left(V_{l}, E_{l}\right)$. Let us assume that both $G_{p}$ and $G_{l}$ satisfy the necessary conditions (stated in Section II) for the existence of a survivable routing. For each virtual link $(s, t)$ in the virtual topology, we need to compute a path in the physical topology. Let $f_{i j}^{s t}=1$ if the virtual link between $(s, t)$ is routed over the physical link $(i, j)$ and 0 otherwise. Let $g_{r}^{s t}=1$ if the virtual link between $s, t$ is routed over at least one of the physical links that belongs to the SRLG set $r$. Let $h_{i}^{s t}=1$ if the virtual link $(s, t)$ is routed through physical node $i$. Our new enhanced ILP formulation for SRLG and node failures (EMOD-SN) is built upon the ILP formulation (MOD-L) (presented in [2]) by enhancing the survivability constraints given by Eq. 7 and 8. Our EMOD-SN ILP formulation also considers wavelength assignment. 
The objective of the EMOD-SN ILP formulation is to minimize the total number of wavelength-links used for survivable routing. The EMOD-SN ILP formulation follows:

Minimize

$$
\sum_{\substack{(i, j) \in E_{p} \\(s, t) \in E_{l}}} f_{i j}^{s t}
$$

Subject to connectivity constraints: $\forall(s, t) \in E_{l}, \forall i \in V_{p}$

$$
\sum_{\text {js.t. }(i, j) \in E_{p}} f_{i j}^{s t}-\sum_{\text {js.t. }(j, i) \in E_{p}} f_{j i}^{s t}=\left\{\begin{aligned}
1, & \text { if } \mathrm{s}=\mathrm{i} \\
-1, & \text { if } \mathrm{t}=\mathrm{i} \\
0, & \text { otherwise }
\end{aligned}\right.
$$

The connectivity is modeled as a multi-commodity flow. Equation 2 gives the connectivity constraints of routing one unit of flow from node $s$ to $t$.

Virtual-edge and SRLG conflict constraint: $\forall r \in R_{p}$ and $\forall(s, t) \in E_{l}$

$$
\begin{aligned}
g_{r}^{s t} & \leq \sum_{\forall(i, j) \in r} f_{i j}^{s t} \\
\left|E_{p}\right| * g_{r}^{s t} & \geq \sum_{\forall(i, j) \in r} f_{i j}^{s t}
\end{aligned}
$$

Node and Virtual-edge conflict constraint: $\forall i \in V_{p}$ and $\forall(s, t) \in E_{l}$

$$
\begin{aligned}
h_{i}^{s t} & \leq \sum_{\forall(i, j) \in E_{p}} f_{i j}^{s t}+\sum_{\forall(k, i) \in E_{p}} f_{k i}^{s t} \\
\left|E_{p}\right| * h_{i}^{s t} & \geq \sum_{\forall(i, j) \in E_{p}} f_{i j}^{s t}+\sum_{\forall(k, i) \in E_{p}} f_{k i}^{s t}
\end{aligned}
$$

SRLG survivability constraints: $\forall r \in R_{p}$ and $\forall$ primary cutset $\left\langle S, V_{l} \backslash S\right\rangle$

$$
\sum_{(s, t) \in\left\langle S, V_{l} \backslash S\right\rangle} g_{r}^{s t}<\left|\left\langle S, V_{l} \backslash S\right\rangle\right| .
$$

Equation 7 gives the survivability constraints under single SRLG failures which is a direct consequence of Theorem 2. Only primary cut-sets of the virtual topology are considered instead of all cut-sets. This reduces the number of constraints and results in improved performance in terms of time.

Node survivability constraints: $\forall G_{l, i}, i \in V_{p}$ and $\forall P_{G_{l, i}}\left\langle S, V_{l, i} \backslash S\right\rangle$

$$
\sum_{(s, t) \in P\left\langle S, V_{l} \backslash S\right\rangle} h_{i}^{s t}<\left|P\left\langle S, V_{l, i} \backslash S\right\rangle\right| .
$$

Equation 8 gives the survivability constraint against single node failures which is a direct consequence of Theorem 5 .

Wavelength assignment constraints: $\forall(s, t) \in E_{l}$

$$
\sum_{1 \leq w \leq W} \lambda^{s t}(w)=1
$$

$\lambda^{s t}(w)=1$ if wavelength $w$ is assigned along the physical route for the virtual link $(s, t)$ otherwise 0 . Constraint 9 states that one and only one wavelength is assigned to physical path corresponding to every logical link in the virtual topology. $\lambda_{i j}^{s t}(w)=1$ if wavelength $w$ is assigned to link $(i, j)$ in physical topology for routing the virtual link $(s, t)$ otherwise 0 . Since no wavelength conversion is assumed the wavelength continuity constraint is followed.
Wavelength usage constraints: $\forall(i, j) \in E_{p}, 1 \leq w \leq W$

$$
\sum_{(s, t) \in E_{l}} \lambda_{i j}^{s t}(w)+\lambda_{j i}^{s t}(w) \leq 1
$$

Constraint 10 states that a wavelength can be used at most once on any given link in the physical topology. Capacity constraints: $\forall(i, j) \in E_{p}$

$$
\sum_{(s, t) \in E_{l}} f_{i j}^{s t}+f_{j i}^{s t} \leq W
$$

The capacity constraint 11 states that the total number of logical links that physical link $(i, j)$ can support is at most $W$. Constraints on $\lambda_{i j}^{s t}(w): \forall(s, t) \in E_{l}, \forall(i, j) \in E_{p}, w \in$ $\{1 \ldots W\}$

$$
\begin{gathered}
\lambda_{i j}^{s t}(w)+\lambda_{j i}^{s t}(w) \leq \lambda^{s t}(w) \\
\lambda_{i j}^{s t}(w)+\lambda_{j i}^{s t}(w) \geq \lambda^{s t}(w)+f_{i j}^{s t}+f_{j i}^{s t}-1 \\
\lambda_{i j}^{s t}(w)+\lambda_{j i}^{s t}(w) \leq f_{i j}^{s t}+f_{j i}^{s t}
\end{gathered}
$$

Integer constraints:

$$
\lambda_{i j}^{s t}(w), \lambda^{s t}(w), f_{i j}^{s t} \in\{0,1\}
$$

Constraint 12 states that if wavelength $w$ is not assigned to the logical link between nodes $s$ and $t$ then wavelength $w$ is not assigned on any physical link for routing this logical link. Constraint 13 states that if logical link $(s, t)$ is routed on physical link $(i, j)$ or $(j, i)$ and $w$ is the wavelength assigned to the logical link $(s, t)$ then wavelength $w$ is reserved along the $(i, j)$ or $(j, i)$ respectively for logical link $(s, t)$. Constraint 14 states that wavelength $w$ is reserved along link $(i, j)$ or $(j, i)$ for logical link $(s, t)$ if the logical link $(s, t)$ is routed along the link $(i, j)$ or $(j, i)$ respectively. Constraint 15 states that all the variable are binary variables.

We present the results of computing survivable routing by solving our EMOD-SN ILP formulation in Section V. The results show that the size of the EMOD-SN ILP for problem instances where the virtual topology is a general graph, even for medium sized physical topologies, becomes too large to solve. The size of the EMOD-SN formulation is directly proportional to exponential number of primary cut-sets of the general graph virtual topology. In the next section we introduce sub-classes of graphs that have polynomial number of primary cut-sets.

\section{Hierarchical Planar CyCles as Virtual TOPOLOGIES}

In the earlier section we presented an ILP formulation to the survivable routing problem. But when the virtual topology graph is a general graph the number of primary cuts of a graph grows exponentially with the number of nodes in the graph. Our experimental results presented in Section V show that the survivable routing of general graph virtual topologies on EUROPEAN network physical topology with 19 nodes could not be computed (within a 2 hour time limit) by solving the EMOD-SN ILP formulation. In this section we introduce sub-classes of graphs, called planar cycles and hierarchical planar cycles, that have polynomial number of primary cuts. In a simple-cycle, any pair of edges is a primary cut and 


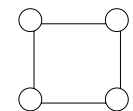

(a)

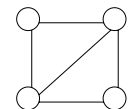

(b) (c)

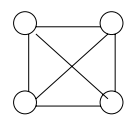

Fig. 3. Examples of planar cycles and non-planar cycles.
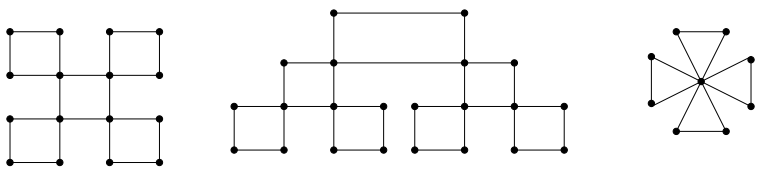

Fig. 4. Examples of hierarchical cycle graphs.

every primary cut-set has exactly two edges. Therefore the number of primary cuts in a simple-cycle is $\left(\begin{array}{l}n \\ 2\end{array}\right)=n(n-1) / 2$. A cycle has an edge-connectivity of 2 and its average hop length is $n / 2$ which is directly proportional to the size of the cycle. These disadvantages hinder the usability of cycles as virtual topologies in real networks. Besides, due to its low connectivity it is more difficult to compute a survivable routing. In this section we present three sub-classes of graphs that not only have polynomial number of cut-sets but also have better connectivity. Using these classes of graphs as virtual topologies, it is not only easier to compute a survivable routing by solving ILP formulation but also these topologies support a mesh-like traffic demand pattern.

Let us present these sub-classes of graphs. A graph is planar if it has a drawing without crossings [13]. A drawing of a graph is a planar embedding of the graph on a plane.

Definition 7: Planar cyclic graph: A graph is called planar cyclic graph if it has a drawing of a simple cyclic graph connecting all the vertices and having chords that do not cross.

In the rest of the document we will refer to planar cyclic graphs as planar cycles. If $C$ is a planar cycle, then it has a drawing $D_{C}$ called planar drawing where edges do not intersect. If $D_{C}$ is a planar drawing of a planar cycle, then the edges along the longest cycle in the drawing $D_{C}$ are called cycle edges. The non-cycle edges of the $D_{C}$ are called chords. Figures 3(a) and 3(b) are examples of planar cycles while Figure 3(c) is an example of a non-planar cycle. The following observation is very interesting.

Lemma 8: Addition of edges to a graph $G$ does not decrease number of primary cuts in $G$. (For proof, please refer to [17]).

Theorem 9: A planar cycle has the same number of primary cuts as a simple cycle of same size. (For proof, please refer to [17]).

From Theorem 9 it follows that the number of primary cuts of a planar cycle is $n(n-1) / 2$ where $n$ is the number of nodes. The number of primary cut-sets of a simple cycle do not increase by adding chords to the cycle that do not intersect. But it may increase the number of edges in some cut-sets of the cycle. The maximum number of chords that can be added to a simple cycle such that resulting cycle is a planar cycle is $n-3$. Therefore the maximum number of edges in a planar cycle of size $n$ is $2 n-3$. A planar cycle with $2 n-3$ edges is called a complete planar cycle.

A hierarchical cycle is a collection of cycles such that
TABLE I

NUMBER OF PRIMARY CUT-SETS FOR NETWORKS OF VARYING SIZES.

\begin{tabular}{|c|c|c|c|}
\hline No. Nodes & General graph & SC/PC & HC/HPC \\
\hline 10 & 1023 & 45 & 18 \\
\hline 15 & 32767 & 105 & 27 \\
\hline 20 & 1048575 & 190 & 36 \\
\hline
\end{tabular}

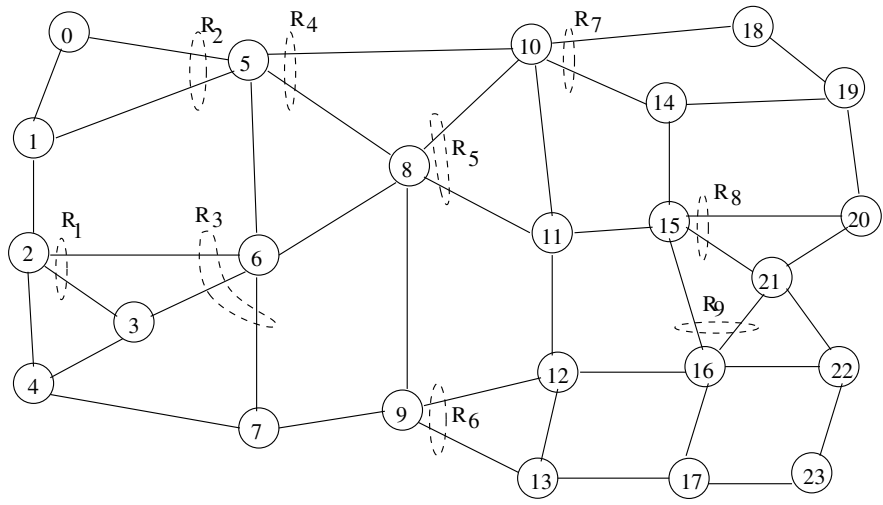

Fig. 5. The 24-node physical network 24-NET.

any two cycles have at most one node in common. Fig. 4 shows examples of hierarchical cycles. A simple cycle is trivially a hierarchical cycle. The hierarchical cycles model the hierarchical self-healing rings [16] . A hierarchical planar cycle is a collection of planar cycles such that any two planar cycles have at most one node in common. The number of primary cut-sets of hierarchical cycles and hierarchical planar cycles are polynomial. Table III gives the number of primary cut-sets for general graphs, SC/PC and $\mathrm{HC} / \mathrm{HPC}$ for varying number of nodes.

Theorem 10: The number of primary cut-sets of a hierarchical cycle graph $H=(V, E)$ consisting of $p$ cycles $C_{1}, C_{2}, \ldots, C_{p}$ are $\left[n_{1}\left(n_{1}-1\right) / 2+n_{2}\left(n_{2}-1\right) / 2+\ldots+\right.$ $\left.n_{p}\left(n_{p}-1\right) / 2\right]$ where $n_{i}$ is the number of nodes in the cycle $C_{i}$ of $H$. (For proof, please refer to [17]).

We conclude that planar and hierarchical planar cycles have polynomial number of primary cut-sets. According to results presented in Section $\mathrm{V}$, the EMOD-SN formulations of survivable routing of virtual topologies belonging to these classes can be solved in reasonable space and time.

\section{EXPERIMENTAL DESIGN AND RESUltS}

We conducted experiments on five different physical network topologies, namely, 10-node mesh network (10-NET), 11-node NJLATA network (NJLATA), 14-node NSF network (NSF), 19-node European network (EURO), and 24-node mesh network (24-NET). The 10-NET has 15 links and 13 SRLGs. The 24-NET physical topology is shown in Figure 5. In our experiments, we used randomly generated degree$k$ regular graphs, $m$-edge general graphs and $m$-edge planar cycles as virtual topologies. An undirected graph is called degree- $k$ regular if all the nodes in the graph have exactly degree $k$. We make sure that the randomly generated graphs satisfy the necessary conditions for the existence of survivable routing. 
TABLE II

ACRONYM TABLE

\begin{tabular}{|r|l|}
\hline Acronym & Expansion \\
\hline \hline$V T$ & Virtual Topology \\
\hline$P T$ & Physical Topology \\
\hline$W$ & Number of Wavelengths \\
\hline$N T$ & No. of Virtual Topologies \\
\hline$A S T$ & No. of Non-Survivable Virtual Topologies \\
\hline$A v g . T i$ & Average Time (in sec) \\
\hline$N-B N W$ & $\begin{array}{l}\text { No. of Blocked topologies due to } \\
\text { No-free Wavelength }\end{array}$ \\
\hline
\end{tabular}

TABLE III

COMPARISON OF RESULTS OF COMPUTING SURVIVABLE ROUTING ON 10-NET, NJLATA AND NSF PHYSICAL TOPOLOGIES USING MOD-S AND EMOD-SN ILP FORMULATIONS.

\begin{tabular}{|c|c|c|c|c|c|}
\hline$I L P$ & $P T$ & $V T T$ & $N T$ & $N S T$ & $\begin{array}{c}\text { Avg. Ti } \\
\text { (in sec) }\end{array}$ \\
\hline \hline MOD-S & $10-$-NET & degree-3 & 500 & 0 & 8.49 \\
\hline EMOD-SN & 10 -NET & degree-3 & 500 & 0 & 3.23 \\
\hline MOD-S & $10-$-NET & 15 -edge & 300 & 18 & 15.22 \\
\hline EMOD-SN & $10-$ NET & 15 -edge & 300 & 18 & 4.43 \\
\hline MOD-S & NJLATA & 20-edge & 800 & 0 & 26.2 \\
\hline EMOD-SN & NJLATA & 20-edge & 800 & 0 & 9.53 \\
\hline MOD-L & NSF & 21-edge & 100 & 2 & 424 \\
\hline EMOD-SN & NSF & 21-edge & 100 & 2 & 23 \\
\hline
\end{tabular}

We experimented with EMOD-SN and MOD-L ([2]) ILP formulations. For comparison in SRLG graphs, we use MOD-S, which is a simple modification of EMODSN to consider both primary and secondary cuts. The ILP formulations are solved using ILOG CPLEX software package on Sun Sparc Ultra-60 workstation. The performance of the ILP formulations is measured in terms of the number of wavelength-links used for survivable routing and the time taken to compute the survivable routing. Table II lists the acronyms used in the rest of the paper. The number of topologies for which survivable routing does not exist is $\mathrm{N}$ NSVT. The acronym Avg. Ti represents the average time taken to compute the survivable routing of a virtual topology. The acronym $N-B N W$ represents the number of blocked survivable routings due to no-free wavelength.

Table III presents the results of computing survivable routing of degree-3 and 15-edge virtual topologies on 10NET physical topology and 20-edge virtual topologies on NJLATA using EMOD-SN and MOD-S ILP formulations. In our experiments, all the links of 10-NET, NJLATA and NSF physical topologies have 4, 6 and 8 wavelengths respectively. Survivable routing was computed for all the 500 degree3 virtual topologies on 10-NET physical topology. Among the 300 15-edge virtual topologies survivable routing does not exist for 18 topologies. The average time taken for computing survivable routing by EMOD-SN ILP is less than that of average time taken by MOD-S ILPs. Table IV shows the number of survivability constraints for ILP formulations
TABLE IV

NUMBER OF CONSTRAINTS OF ILP FORMULATIONS FOR DIFFERENT PHYSICAL AND VIRTUAL TOPOLOGIES.

\begin{tabular}{|c|c|c|c|}
\hline ILP & PT & VT & Constraints \\
\hline \hline EMOD-SN & EURO & degree-3 & 32665 \\
\hline MOD-L & EURO & degree-3 & 10223600 \\
\hline EMOD-SN & $10-N E T$ & degree-3 & 2830 \\
\hline MOD-L & 10-NET & degree-3 & 9709 \\
\hline EMOD-SN & NJLATA & 20 -edge & 6083 \\
\hline MOD-L & NJLATA & 20-edge & 18414 \\
\hline
\end{tabular}

TABLE V

RESULTS OF COMPUTING SURVIVABLE ROUTING OF M-EDGE PLANAR CYCLE (PC) VIRTUAL TOPOLOGIES USING EMOD-SN ILP FORMULATION.

\begin{tabular}{|c|c|c|c|c|c|}
\hline$P T$ & $V T$ & $N T$ & $N S T$ & $\begin{array}{c}A v g . ~ T i \\
\text { (in sec })\end{array}$ & $\begin{array}{c}\text { Max. Ti } \\
\text { (in sec) }\end{array}$ \\
\hline \hline NSF & 20-edge PC & 100 & 24 & 2.25 & 8.6 \\
\hline NSF & 25-edge PC & 100 & 0 & 2.474 & 43.6 \\
\hline EURO & 30 -edge PC & 100 & 4 & 17.5 & 63.7 \\
\hline EURO & 35 -edge PC & 100 & 0 & 16.7 & 2875.3 \\
\hline 24-NET & 40 -edge PC & 100 & 7 & 120.0 & 350.5 \\
\hline 24-NET & 45 -edge PC & 100 & 0 & 112.0 & 279.7 \\
\hline
\end{tabular}

MOD-L/MOD-S and EMOD-SN for 10-NET, NJLATA and EURO physical topologies and randomly generated general graph virtual topology (as stated in Section II).

Using MOD-L ILP, we computed the survivable routing of general graph virtual topologies on the 14 node NSF network. The survivable routing for general graph virtual topologies on EURO and 24-NET physical topologies could not be computed (within 2 hour time limit) even after using our enhanced EMOD-SN ILP formulation. Table V shows the result of computing survivable routing of $m$-edge planar cycle virtual topologies on NSF, EURO and 24-NET physical networks of sizes 14, 19 and 24 respectively. The average time taken to compute survivable routing of 40-edge planar cycle virtual topologies on 24-NET physical network is 2 min. The maximum time taken to compute survivable routing of 35-edge planar cycle virtual topologies on EURO physical network is $50 \mathrm{~min}$. These results show that using planar cycle virtual topologies survivable routing can be computed using our enhanced EMOD-SN formulation even on large physical networks.

\section{CONCLUSIONS}

In this work we studied the problem of survivable routing of a virtual topology on a physical topology under SRLG/node failures. We presented a classification of cuts of a graph into primary and secondary cuts and proved that the survivable routing need only consider primary cuts of a graph. Based on this result, we presented an enhanced ILP formulation, EMOD-SN, to solve the survivable routing problem under SRLG and node failures. The EMOD-SN ILP formulation attains $3-18$ times speed-up in comparison to the earlier 
formulation, MOD-L. We introduced sub-classes of graphs called hierarchical planar cycles that have polynomial number of cut-sets. Using the enhanced EMOD-SN ILP formulation and planar cycles as virtual topologies, the survivable routing could be computed for medium or large sized networks, thus increasing the scalability. In our experiments, we computed survivable routing of 45 -edge planar cycle virtual topologies on physical topologies of sizes up to 24 nodes with the average computation time of $2 \mathrm{~min}$ and maximum computation time of $6 \mathrm{~min}$.

\section{REFERENCES}

[1] J. Strand, A. L. Chiu, and R. Tkach, "Issues for routing in the optical layer," IEEE Commun. Mag., vol. 39, no. 2, pp. 81-87, Feb. 2001.

[2] E. Modiano and A. Narula-Tam, "Survivable lightpath routing: a new approach to the design of WDM-based networks," IEEE J. Sel. Areas Commun., vol. 20, no. 4, pp. 800-809, May 2002.

[3] D. Banerjee and B. Mukherjee, "A practical approach for routing and wavelength assignment in large wavelength-routed optical networks," IEEE J. Sel. Areas Commun. vol. 14, no. 5, pp. 903-908, June 1996.

[4] R. Ramaswami and K. N. Sivarajan, "Design of logical topologies for wavelength-routed optical networks," IEEE J. Sel. Areas Commun. vol. 14, no. 5, pp. 840-851, June 1996.

[5] R. Dutta and G. N. Rouskas, "A survey of virtual topology design algorithms for wavelength routed optical networks," Opt. Networks Mag., vol. 1, no. 1, pp. 73-89, Jan. 2000.

[6] O. Crochat and J-Y. L. Boudec, "Design protection for WDM optical networks," IEE J. Sel. Areas Commun., vol. 16, no. 7, Sept. 1998.

[7] A. Sen, B. Hao, B. H. Shen, and G. H. Lin, "Survivable routing in WDM networks: a logical ring in arbitrary physical topology," in Proc. IEEE International Communication Conference ICC '02, New York, June 2002.

[8] H. Lee, H. Choi, S. Subramaniam, and H. A. Choi, "Survival embedding of logical topology in WDM ring networks," in Proc. Symposium on Photonics, Networking and Computing, The 6th Joint Conference on Information Sciences (JCIS), Duke University, March 2002.

[9] H. Lee, H. Choi, S. Subramaniam, and H. A. Choi, "Survival logical topology design in WDM optical ring networks," in Proc. 39th Allerton Conference on Communications, Control, and Computing, Univ. of Illinois at Urbana-Champaign, Oct. 2001.

[10] M. Maliosz, and T. Cinkler, "Configuration of protected virtual private networks," DRCN 2001, Budapest, Hungary, Oct. 2001.

[11] F. Giroire, A. Nucci, N. Taft, C. Diot, "Increasing the robustness of IP backbones in the absence of optical level protection," in Proc. IEEE INFOCOM, San Francisco, 2003.

[12] I. P. Kaminow and T. L. Koch, Eds., Optical Fiber Telecommunications IIIA. Academic Press, 1997.

[13] D. B. West, Introduction to Graph Theory, Second Edition. Prentice Hall, 1996.

[14] M. R. Garey and D. S. Johnson, Computers and Intractability: A Guide to the Theory of NP-Completeness. W. H. Freeman and Company.

[15] J. Y. Yen, "Finding the $\mathrm{k}$ shortest loopless paths in a network," Management Science, vol. 17, pp. 712-716, 1971.
[16] J. Shi and J. P. Fonseka, "Hierarchical self-healing rings," IEEE/ACM Trans. Netw., vol. 3, no. 6, Dec. 1995.

[17] A. Todimala, "Fault-tolerance using shared path protection in wavelength division multiplexing optical transport networks," Ph.D. thesis, University of Nebraska - Lincoln, Department of Computer Science and Engineering, 2006.

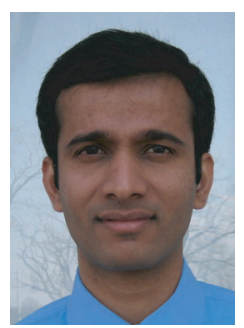

Ajay Kumar Todimala received his BE degree in Computer Science and Engineering from Maturi Venkata Subba Rao Engineering College, Osmania University, Hyderabad (India) in 2000. He received his M.S. and Ph.D degrees in Computer Science from University of Nebraska, Lincoln in 2003 and 2006, respectively.

$\mathrm{He}$ is faculty research assistant at Mid Atlantic Crossroads (MAX), University of MarylandCollege park. He served as a reviewer for IEEE INFOCOM, ICC, GLOBECOM conferences and IEEE Transactions on Networking. His research interests include design of optical WDM networks, network survivability, design and analysis of algorithms. His email address is: ajay.todimala@gmail.com.

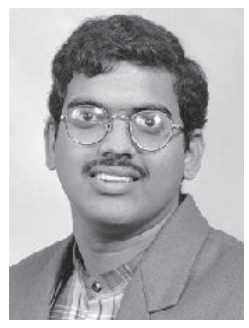

Byrav Ramamurthy received his B.Tech. degree in Computer Science and Engineering from Indian Institute of Technology, Madras (India) in 1993. He received his M.S. and Ph.D. degrees in Computer Science from University of California (UC), Davis in 1995 and 1998, respectively.

Since August 2003, he has been an associate professor in the Department of Computer Science and Engineering at the University of NebraskaLincoln (UNL). He was the Feature Editor on Theses for the Optical Network Magazine. He served as a guest co-editor for a special issue of IEEE Network Magazine on Optical Communication Networks. He served as a member of the technical program committees for the IEEE INFOCOM, IEEE GLOBECOM, Opticomm/Broadnets, ICC and ICCCN conferences. He is author of the book Design of Optical WDM Networks - LAN, MAN and WAN Architectures and a co-author of the book Secure Group Communications over Data Networks, published by Kluwer Academic Publishers/Springer in 2000 and 2004 respectively. From 2001-2003, he served as the founding secretary of the IEEE ComSoc Optical Networking Technical Committee (ONTC) for which he currently serves as the Secretary and Online Content Chair. He served as the TPC Co-chair for the IEEE ICC 2006 Optical Systems and Networks Symposium and the Broadnets 2006 Optical Symposium. He serves as the INFOCOM 2008 Miniconference TPC Co-chair and as the INFOCOM 2008 EDAS Chair.

Prof. Ramamurthy was a recipient of the Indian National Talent Search scholarship and was a fellow of the Professors for the Future program at UC Davis. He is a recipient of the UNL Research Council Grant-in-Aid award for 1999, the College of Engineering and Technology Faculty Research Award for 2000 and the UNL CSE Dept. Student Choice Outstanding Teaching Award for Graduate-level Courses for 2002-2003 and 2006-2007. His research areas include optical networks, wireless/sensor networks, network security, distributed computing, and telecommunications. His research is supported by the U.S. National Science Foundation, Agilent Tech., and OPNET Inc. His email address is: byrav@cse.unl.edu. 